\title{
A refined radio-telemetry technique to monitor right ventricle or pulmonary artery pressures in rats: a useful tool in pulmonary hypertension research
}

\author{
M. L. Handoko • I. Schalij • K. Kramer • A. Sebkhi • \\ P. E. Postmus - W. J. van der Laarse - W. J. Paulus • \\ A. Vonk-Noordegraaf
}

Received: 10 May 2007 /Revised: 21 July 2007 / Accepted: 16 August 2007 / Published online: 2 October 2007

(C) Springer-Verlag 2007

\begin{abstract}
Implantable radio-telemetry methodology, allowing for continuous recording of pulmonary haemodynamics, has previously been used to assess effects of therapy on development and treatment of pulmonary hypertension. In the original procedure, rats were subjected to invasive thoracic surgery, which imposes significant stress that may disturb critical aspects of the cardiovascular system and delay recovery. In the present study, we describe and compare the original trans-thoracic approach with a new, simpler trans-diaphragm approach for catheter placement, which avoids the need for surgical
\end{abstract}

Electronic supplementary material The online version of this article (doi: 10.1007/s00424-007-0334-z) contains supplementary material, which is available to authorized users.

M. L. Handoko $(\bowtie) \cdot$ W. J. van der Laarse $\cdot$ W. J. Paulus Department of Physiology, Institute for Cardiovascular Research, VU University Medical Center,

Van der Boechorststraat 7,

1081 BT Amsterdam, The Netherlands

e-mail: ml.handoko@vumc.nl

M. L. Handoko - I. Schalij · P. E. Postmus · A. Vonk-Noordegraaf Department of Pulmonology,

VU University Medical Center,

Amsterdam, The Netherlands

K. Kramer

Department of Health, Safety and Environmental Affairs,

VU University,

Amsterdam, The Netherlands

A. Sebkhi

Section on Clinical Pharmacology, Faculty of Medicine, Imperial College, Hammersmith Hospital,

London, UK invasion of the thorax. Satisfactory overall success rates up to $75 \%$ were achieved in both approaches, and right ventricular pressures and heart and respiratory rates normalised within 2 weeks. However, recovery was significantly faster in trans-diaphragm than in transthoracic operated animals $(6.4 \pm 0.5$ vs $9.5 \pm 1.1$ days, respectively; $p<0.05)$. Stable right ventricular pressures were recorded for more than 4 months, and pressure changes, induced by monocrotaline or pulmonary embolisms, were readily detected. The data demonstrate that right ventricular telemetry is a practicable procedure and a useful tool in pulmonary hypertension research in rats, especially when used in combination with echocardiography. We conclude that the described trans-diaphragm approach should be considered as the method of choice, for it is less invasive and simpler to perform.

Keywords Blood pressure $\cdot$ Heart · pulmonary artery · Diaphragm · Hypertensive rats · Cardiac output .

Power output - Vascular remodelling · Circulation .

Respiration

\section{Introduction}

Over the last decade, new medical treatments became available for the treatment of pulmonary hypertension $(\mathrm{PH})$ patients, and this development set off a renewed interest in the pathophysiology and haemodynamics of the pulmonary circulation [6, 12, 22]. Experimental PH studies are still largely confined to single measurements of pulmonary arterial (PA) or right ventricular (RV) pressure in anaesthetised, open-chest animals undergoing artificial respiration, which does not allow determining changes of 
pressures in time. Measuring pulmonary haemodynamics have improved considerably since RV telemetry was firstly introduced by Hess et al. [4], as it allows for continuous monitoring of RV or PA pressures, without introducing artefacts caused by stress or anaesthetics [9]. Moreover, animals fitted with telemetry can serve as their own controls, thus permitting the use of smaller groups of animals than is possible with the single measurement approach in anaesthetised animals [9]. Although many investigators have embraced this new technology with enthusiasm and despite its proven potential, studies actually using this methodology in rats are scarce $[4,13,16,18,19]$.

One possible explanation for this discrepancy is that the original procedure was only described briefly before [4], complicating its implementation for potential new users. Secondly, the described trans-thoracic approach involves RV catheterisation through complex and highly invasive thoracotomy [4]. In the present study, we hypothesised that RV catheterisation performed via an opening in the diaphragm, thereby omitting a thoracotomy, may be a simpler, less invasive alternative.

In the present report, we provide a detailed description of the alternative trans-diaphragm approach together with a comparison with the original trans-thoracic approach. Furthermore, we illustrate its usefulness when studying the pulmonary circulation, by presenting novel comprehensive pulmonary and cardiac haemodynamic data during the development of monocrotaline-induced pulmonary hypertension.

\section{Materials and methods}

All experiments were approved by the Institutional Animal Care and Use Committee of the VU University and were conducted in accordance with the European Convention for the Protection of Vertebrate Animals used for Experimental and Other Scientific Purposes, and the Dutch Animal Experimentation Act.

Animals

Thirty-eight male Wistar rats were used (250-300 g; Harlan, Horst, The Netherlands), of which 33 were operated and 5 served as controls (no surgery). The animals were allowed to adapt to their new environment for at least 1 week before surgery. The animals were conventionally housed in pairs under controlled conditions (temperature $21-22^{\circ} \mathrm{C}$; humidity $60-65 \% ; 12: 12 \mathrm{~h}$ light-dark cycle) and had free access to filtered water and standard rat chow (Global 2016, Harlan Teklad, Blackthorn-Bicester, England).
Telemetry system

An implantable radio-telemetry system for blood pressure measurements for small laboratory animals was used, comprising a radio-transmitter (TA11PA-C40) fitted with a 10-cm-long catheter (Data Science International [DSI], St. Paul, MN). Transmitters were turned on a day before implantation and stored in sterilised saline, following the manufacturer's instructions (http://www.datasci.com/ information/index.asp).

The pressure was monitored continuously during surgery to ensure proper position of the tip of the pressure catheter. After successful implantation of the transmitter, RV or PA pressures and locomotor activity were recorded for $10 \mathrm{~s}$ every $5 \mathrm{~min}$. From the pressure recordings mean, systolic and diastolic pressure, heart rate, respiratory rate and circadian rhythm were subsequently derived (Dataquest A. R.T. software 4.0, DSI).

Pre-operative care and anaesthesia

All animals received pre-surgical intramuscular buprenorphine analgesia $(0.10 \mathrm{mg} / \mathrm{kg}$; Schering-Plough, Maarssen, The Netherlands). For general anaesthesia, isoflurane $(2.0 \%$ in 1:1 $\mathrm{O}_{2}$ /air mix; Pharmachemie, Haarlem, The Netherlands) was used via induction chamber and tracheal intubation $(16 \mathrm{G} \times 51-\mathrm{mm}$ Teflon tube; ventilator settings: breathing frequency $80 / \mathrm{min}$, pressures: $9 / 0 \mathrm{cmH}_{2} \mathrm{O}$, inspiratory/expiratory ratio 1:1). For extra local analgesia, lidocaine spray $(100 \mathrm{mg} / \mathrm{ml}$; AstraZeneca, Zoetermeer, The Netherlands) was applied at the surgical region. Animals were maintained under anaesthesia for an average time of $80 \mathrm{~min}$.

Animals were placed on a heating pad to maintain body temperature and positioned in dorsal recumbency. Hydromellose drops $(0.3 \%$; Ratiopharm, Zaandam, The Netherlands) were applied to prevent drying of the eyes. After shaving and disinfection with $70 \%$ ethanol of the chest and abdomen, animals were covered with sterile incision foil and the animals were then covered with sterile incision foil (Opraflex, Lohmann \& Rauscher, Almere, The Netherlands). A surgical microscope was used (magnification $\times 16-64$; Carl Zeiss, Sliedrecht, The Netherlands) for optimal view.

Surgery: trans-thoracic approach

\section{Implantation of the telemetry transmitter in the abdomen}

The abdominal cavity was accessed via a 5 -cm midline laparotomy, starting just below the xiphoid process. The transmitter was placed in the peritoneal cavity, with the catheter pointing caudally to prevent liver injury and tissue 
reaction. The abdominal cavity was covered with gauzes soaked in warm saline and left open until the end of the procedure.

\section{Routing of the pressure catheter to the right ventricle}

The heart was exposed by means of a left thoracotomy performed at the sixth intercostal space and mid-clavicular line. The thorax was opened with blunt scissors, respecting the anatomy of the overlying muscle layers and cautiously avoiding injury to the lungs. The pressure catheter was then tunnelled subcutaneously from the peritoneal cavity to the opening in the thorax and temporarily laid aside. Four individual small hooks were used to retract the ribs and expose the heart through a wider, $2 \times 2-\mathrm{cm}$ window. The pericardium was then opened with two dressing forceps.

\section{Catheterisation of the right ventricle}

A superficial purse string (6-0 Prolene, Ethicon, StStevens-Woluwe, Belgium) was placed on the right ventricle free wall, near the apex. Through the purse string, the right ventricle was punctured with a $19 \mathrm{G}$ syringe needle in the direction of the RV outflow tract, avoiding any coronary vessel. After removing the needle, the site was wiped with a sterile cotton stick. The tip of the catheter was then carefully inserted into the right ventricle, using a vessel canulation forceps $(0.5-1.0 \mathrm{~mm}$ outer diameter, Fine Science Tools, Heidelberg, Germany), avoiding loss of gel from the tip at all times. Live pressure waveform trace confirmed proper RV catheterisation (RV systolic/diastolic pressures are typically $\sim 25 / 1 \mathrm{mmHg}$ ). Depending on the protocol, the pressure catheter was subsequently advanced for about $2 \mathrm{~cm}$ more, positioning the tip of the catheter beyond the pulmonary valves. Again, live pressure waveform trace confirmed correct positioning of the pressure catheter in the pulmonary artery (PA systolic/diastolic pressures are typically $\sim 25 / 10 \mathrm{mmHg}$; Fig. 1, left panel). Finally, the catheter was fixed by the purse string and a small drop of tissue adhesive at the site of insertion (10 $\mu$ l dispensed by a pipette; Vetbond, 3M, St. Paul MN; Electronic Supplementary Material (ESM) Fig. E-1a).

\section{Closing of the chest and abdomen}

After the catheterisation, blood clots if any were removed from the pleural cavity with a moist cotton stick. The ribs were approximated with two individual sutures (5-0 Vicryl, Ethicon). To promote full expansion of the lungs, a small burst of positive pressure was applied (maximally $10 \mathrm{cmH}_{2} \mathrm{O}$ for $1 \mathrm{~s}$ ). A chest tube $(18 \mathrm{G})$, used as air outlet, was removed after proper thorax excursions were observed. The chest muscles were laid back in layers (no suturing necessary). Then, the transmitter was sutured to the abdominal muscle (5-0 Perma-Hand, Ethicon) incorporating the suture rib of the device. The abdominal wall was sutured in a running subcuticular pattern (5-0 Vicryl). Finally, the skin of the chest and abdomen was closed with a running suture (5-0 Vicryl).

Surgery: trans-diaphragm approach

\section{Opening of abdomen and the diaphragm}

The abdominal cavity was accessed via a 5 -cm midline laparotomy, with the abdominal wall retracted by two individual hooks. To expose the diaphragm, a retraction suture was used to lift the xiphoid process during the whole procedure. The visceral organs were covered temporarily with wet gazes, and the liver was gently pushed down by the weight of a blade holder. A small midline incision in the diaphragm was then made, with small sharp scissors, from the xiphoid process until the tendinous part of the diaphragmatic membrane. The two sides of the diaphragm were held aside with two retraction sutures, providing optimal exposure of the heart (ESM Fig. E-1b).

\section{Catheterisation of the right ventricle}

RV catheterisation was performed similarly, as described above. In short, the pericardium was opened by two dressing forceps, and a purse string was placed just right of the apex. The pressure catheter was then inserted into the right ventricle through a small puncture made with a syringe needle and fixed in place.

\section{Closing the diaphragm and abdomen}

After catheterisation, blood clots in the thorax, if any, were removed. The diaphragm was closed with a running suture (5-0 Vicryl), starting from the ventral side, with the pressure catheter sticking out the diaphragm at the dorsal end of the incision. After that, the retraction suture through the xiphoid process was removed. To promote full expansion of the lungs, a small burst of positive pressure was applied, as described above, with the chest tube between the sutures of the diaphragm, until proper thorax excursions were visually confirmed. To further secure closure of the diaphragm, a small amount of tissue adhesive (Vetbond, 3M) was applied. Finally, the transmitter was implanted in the peritoneal cavity, fixed to the abdominal wall with the catheter facing caudally, and the abdomen was closed, as described above. 
Post-operative care

To compensate for loss of fluids, all animals received warm sterile saline at the end of the procedure $(5 \mathrm{ml}$ intraperitoneal). After final skin closure, the animals were allowed to regain consciousness.

In the first $24 \mathrm{~h}$ of recovery, the animals were housed in individual cages. Each cage was placed halfway on a heating pad, in such a way that half of the cage was maintained above it and the other at room temperature. During this period, the animals were monitored three times, received post-surgical analgesia when clinically indicated (buprenorphine $0.10 \mathrm{mg} / \mathrm{kg}$ subcutaneous) and were provided with drinking gel pads and softened rat chow. After $24 \mathrm{~h}$, the animals were conventionally housed in pairs for a full recovery, and they were inspected and weighted daily. Animals were considered fully recovered from surgery when their appearance and behaviour were normal, their surgical wounds healed, their pre-surgical body weight regained, when the pressures normalised and circadian rhythm was restored. The study period was ended after 4 months.

\section{Experimental protocol}

To induce acute and chronic pulmonary hypertension, four animal received an intravenous bolus injection of microspheres [25] (tail; 1.5 million /100 g body mass in sterile saline; polystyrene, mean diameter $19 \mu \mathrm{m}$ (\#7520), Duke Scientific, Fremont, CA) and three animals monocrotaline subcutaneous [24] (MCT; $80 \mathrm{mg} / \mathrm{kg}$ in sterile saline; Sigma-Aldrich, Zwijndrecht, The Netherlands), respectively. Experimental interventions were performed no earlier then 2 weeks after the surgery.

\section{RV echocardiography}

After a 2-week recovery, trans-thoracic echocardiographic measurements (ProSound SSD-4000 system equipped with a 13-Mhz linear transducer [UST-5542], Aloka, Tokyo, Japan) were performed on anaesthetised but spontaneously breathing rats (isoflurane $2.0 \%$ in $1: 1 \mathrm{O}_{2}$ /air mix), according to the standards of the American Society of Echocardiography $[10,15]$. To minimise the effects of isoflurane on cardiac function, the time under anaesthesia was set to a maximum of $15 \mathrm{~min}$ [14].

The measurements were compared with untreated rats to check for possible adverse effects related to the procedure. Cardiac output, tricuspid annular plane systolic excursion (TAPSE), RV wall thickness (RVWT) and RV end diastolic diameter (RVEDD) were measured, as described before by Hardziyenka et al. [3]. TAPSE is a parameter for RV systolic function, which is measured in the apical fourchamber view of the heart and expresses the displacement of the lateral tricuspid annulus towards the apex during systole [10].

Estimation of pulmonary vascular resistance and RV power output

Additional echocardiographic measurements were performed in MCT-treated rats and untreated but operated controls, just before the injection, 2 weeks after injection and when the first clinical signs of RV heart failure developed (as indicated by weight loss, dyspnoea and lethargy, after which the rats were euthanised). Pulmonary vascular resistance (PVR) and RV power output (RVpower) were estimated, by combining telemetric pressure data and echocardiographic flow data.

PVR was estimated by Poiseuille's law: $[\mathrm{PVR}] \approx[$ mean $\mathrm{PAP}] /[$ cardiac output $] \approx(0.61 \times[$ systolic $\mathrm{RV}$ pressure $]+$ $2 \mathrm{mmHg}) /[$ cardiac output] [1,23].

RV-power was estimated using a simplified pressurevolume analysis, assuming rectangular pressure-volume loops: $[\mathrm{RV}$-power $] \approx[$ systolic $\mathrm{RV}$ pressure $] \times[$ stroke volume $] \times[$ heart rate $]=[$ systolic $R V$ pressure $] \times[$ cardiac output $]$ $[21,23]$.

\section{Autopsy}

To investigate potential effects of chronic implantation of the telemetry transmitters, the animals were euthanised at the end of the study protocol by exsanguination under isoflurane (4.0\% in 1:1 $\mathrm{O}_{2}$ /air mix). Macroscopic examination of right ventricle, lungs, diaphragm and intercostal muscles were performed, and organ weights (heart, lungs, liver, spleen, kidneys) were measured and compared with controls. Animals treated with MCT or microspheres were excluded from these analyses.

Statistical analyses

All measurements, unless otherwise stated, are presented as mean \pm standard error of the mean. Normal distribution was verified, and Student's $t$ test, analysis of variance or Fisher Exact test were used, when appropriate (SPSS 13.0, SPSS, Chicago IL). A $p$ value less than 0.05 was considered statistically significant.

\section{Results}

Success rate

Seventeen animals were operated using the trans-thoracic approach, and 16 animals were operated using the transdiaphragm approach. 
In the trans-thoracic group, 8 animals showed stable pressure signals for the whole study period of 4 months. Five animals did not recover form surgery, and 4 animals developed instable pressure signals, caused by clot formation inside the pressure catheter within 2 weeks of recovery. Of the 9 failed procedures, 4 occured in the first four attempts.

In the trans-diaphragm group, 9 animals showed stable pressure signal for the whole study period of 4 months. Six animals did not recover form surgery, and one animal developed instable pressure signals, caused by clot formation inside the pressure catheter within 2 weeks of recovery. As in the trans-thoracic group, 4 of the failures occurred in the first four attempts.

By discarding the first 4 animals in each procedure, as we consider this the learning curve, the overall success rate was 8 of $13(62 \%)$ for the trans-thoracic approach and 9 of $12(75 \%)$ for the trans-diaphragm approach.

\section{Recovery from surgery}

Within a week post-surgery, heart and respiratory rates and circadian rhythm normalised in all rats, and no differences were observed between the two approaches (Table 1).
Although all animals regained their pre-surgical body weight within 2 weeks, full recovery was significantly faster in the trans-diaphragm- than the trans-thoracic-operated animals (6.4 \pm 0.5 vs $9.5 \pm 1.1$ days, respectively; $p<0.05$ ).

$\mathrm{RV}$ pressures normalised in all animals within 2 weeks after surgery (RV systolic/diastolic pressures $\sim 25 / 1 \mathrm{mmHg}$ ), irrespective of the method used (Table 1). PA systolic/ diastolic pressures were estimated to be $\sim 25 / 10 \mathrm{mmHg}$ (Fig. 1, left panel). Activity had no significant effect on the stability of the pressure signals.

Echocardiography revealed normal cardiac output $(\sim 110 \mathrm{ml} / \mathrm{min})$, TAPSE $(\sim 3.5 \mathrm{~mm})$, RVWT $(\sim 1.0 \mathrm{~mm})$ and RVEDD $(\sim 3.6 \mathrm{~mm})$ in all rats, and no differences were seen between the differently operated animals and controls (Table 1). Furthermore, other signs of RV dysfunction, like pericardial effusion and tricuspid regurgitation, were not present in any animal.

Acute pressure effects of pulmonary embolisation by microspheres

RV telemetry could readily detect acute changes in RV pressure, induced by a bolus injection of microspheres

Table 1 Main results of this study, all values in mean \pm SEM

\begin{tabular}{|c|c|c|c|}
\hline & Trans-thoracic approach $(n=17)$ & Trans-diaphragm approach $(n=16)$ & Controls $(n=5)$ \\
\hline Success rate ${ }^{a}$ & $8 / 13(62 \%)$ & $7 / 9(79 \%)$ & n.r. \\
\hline \multicolumn{4}{|l|}{ Recovery } \\
\hline $\mathrm{BW}$ to pre-surgical level (days) ${ }^{\mathrm{d}}$ & $9.5 \pm 1.1$ & $6.4 \pm 0.5^{*}$ & n.r. \\
\hline Heart rate $(\mathrm{bpm})^{\mathrm{e}}$ & $362 \pm 4.4$ & $359 \pm 5.7$ & $350-400^{\mathrm{b}}[8,17]$ \\
\hline Respiratory rate $(\mathrm{rpm})^{\mathrm{e}}$ & $90 \pm 2.4$ & $91 \pm 3.8$ & $80-100^{\mathrm{b}}[11,17,20]$ \\
\hline Circadian rhythm $(\text { days })^{\mathrm{e}}$ & $3.4 \pm 0.4$ & $3.7 \pm 0.6$ & n.r. \\
\hline \multicolumn{4}{|l|}{ Pressures $^{\mathrm{e}}$} \\
\hline RVSP (mmHg) & $25 \pm 1.1$ & $24 \pm 0.9$ & $21-26^{\mathrm{b}}[16]$ \\
\hline RVDP (mmHg) & $1.7 \pm 0.3$ & $2.2 \pm 0.2$ & $1-5^{\mathrm{c}}[5]$ \\
\hline \multicolumn{4}{|l|}{ RV echocardiography ${ }^{\mathrm{d}}$} \\
\hline Cardiac output (ml/min) & $107 \pm 4.9$ & $116 \pm 6.9$ & $110 \pm 4.9$ \\
\hline TAPSE $(\mathrm{mm})$ & $3.6 \pm 0.1$ & $3.7 \pm 0.2$ & $3.4 \pm 0.1$ \\
\hline RVWT (mm) & $1.0 \pm 0.1$ & $1.0 \pm 0.1$ & $0.9 \pm 0.1$ \\
\hline RVEDD (mm) & $3.6 \pm 0.1$ & $3.7 \pm 0.1$ & $3.6 \pm 0.1$ \\
\hline \multicolumn{4}{|l|}{ Autopsy ${ }^{\mathrm{f}}$, organ weights (g) } \\
\hline Heart & $1.4 \pm 0.1$ & $1.4 \pm 0.2$ & $1.3 \pm 0.1$ \\
\hline Lungs & $1.5 \pm 0.1$ & $1.5 \pm 0.2$ & $1.5 \pm 0.2$ \\
\hline Liver & $15.4 \pm 0.5$ & $15.0 \pm 0.6$ & $15.1 \pm 0.5$ \\
\hline Spleen & $0.65 \pm 0.03$ & $0.68 \pm 0.04$ & $0.64 \pm 0.04$ \\
\hline Kidneys & $2.4 \pm 0.2$ & $2.3 \pm 0.2$ & $2.3 \pm 0.1$ \\
\hline
\end{tabular}

$n$ Number of animals, n.r. not relevant, $B M$ body mass, RVSP RV systolic pressure, RVDP RV diastolic pressure, TAPSE tricuspid annular plane systolic excursion, $R V W T \mathrm{RV}$ wall thickness, $R V E D D \mathrm{RV}$ end diastolic diameter

$* p<0.05$

${ }^{\text {a }}$ First four attempts were discarded.

${ }^{\mathrm{b}}$ Normal values for Wistar rats, awake and at rest, measured by radio-telemetry

${ }^{\mathrm{c}}$ Normal values for Wistar rats, measured during acute pressure measurements under anaesthesia

${ }^{\mathrm{d}}$ Analyses on surviving animals only

${ }^{\mathrm{e}}$ Analyses on successfully operated animals only (stable signal)

${ }^{\mathrm{f}}$ Analyses on surviving and untreated animals only (no microspheres or monocrotaline) 
Fig. 1 left panel: Live pressure recordings while advancing the pressurecatheter into the pulmonary artery. Notice the stepwise increase in diastolic pressures when manoeuvring the catheter beyond the pulmonary valves (arrow), whereas the systolic pressures remained unchanged. right panel: Live pressure recordings while injecting a bolus injection of microspheres (arrow; $19 \mu \mathrm{m}, 1.5$ million/ 100 g i.v.). Upon embolisation, a significant increase in systolic, diastolic and developed RV pressures was observed. Furthermore, in both recordings, the effect of breathing is noticeable

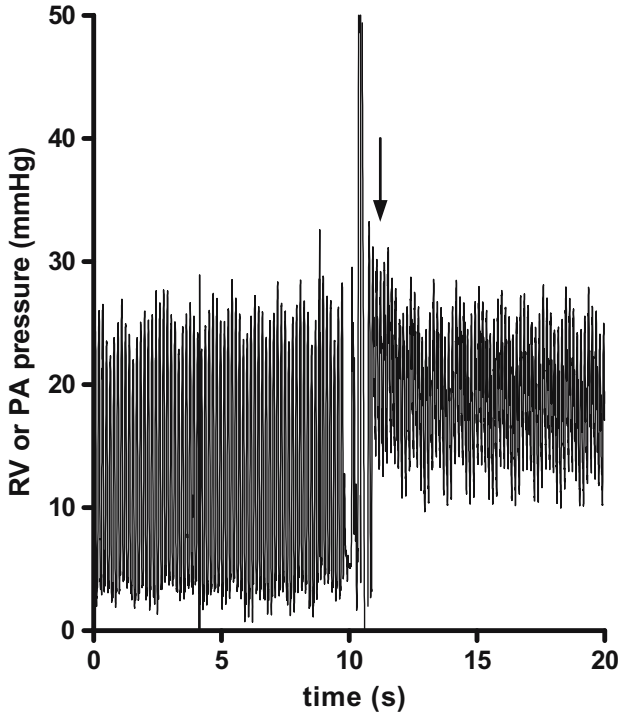

(Fig. 1, right panel). Upon embolisation, systolic RV pressures increased from $21 \pm 1$ to $38 \pm 3 \mathrm{mmHg}(p<0.01)$ and diastolic RV pressures increased from $2 \pm 1$ to $7 \pm$ $2 \mathrm{mmHg}(p<0.05)$.

Haemodynamic changes during the development of MCTinduced pulmonary hypertension

Changes in haemodynamic parameters during the development of MCT-induced PH are shown in Fig. 2 (also in ESM Fig. E-2). Four weeks after MCT injection, the first clinical signs of RV heart failure developed. During this period, there was a gradual rise in RV systolic pressure, from $22 \pm 2 \mathrm{mmHg}$ at baseline to $38 \pm 4 \mathrm{mmHg}$ at 2 weeks and $72 \pm 5 \mathrm{mmHg}$ at 4 weeks $(p<0.01$ vs control). At 4 weeks, cardiac output dropped dramatically, from $100 \pm$ $7 \mathrm{ml} / \mathrm{min}$ at baseline to $107 \pm 8$ at 2 weeks and $52 \pm 4 \mathrm{ml} /$ min at 4 weeks ( $p<0.01$ vs control). In addition, the poor haemodynamic condition of the MCT-treated rats at 4 weeks was confirmed by a simultaneous decline in TAPSE from $3.6 \pm 0.1 \mathrm{~mm}$ during the first 2 weeks to $1.8 \pm$ $0.2 \mathrm{~mm}$ at 4 weeks $(p<0.01$ vs control). During the development of $\mathrm{PH}$, the right ventricle remodelled initially by increasing RVWT $(1.0 \pm 0.1 \mathrm{~mm}$ at baseline, $1.1 \pm$ $0.1 \mathrm{~mm}$ at 2 weeks and $1.3 \pm 0.1 \mathrm{~mm}$ at 4 weeks, respectively; $p<0.01$ vs control) but subsequently by increasing RVEDDs as well $(3.5 \pm 0.1 \mathrm{~mm}$ at baseline, $3.6 \pm 0.1 \mathrm{~mm}$ at 2 weeks and $7.0 \pm 0.5 \mathrm{~mm}$ at 4 weeks, respectively; $p<0.01$ vs control).

During the development of PH, PVR increased continuously, from $1.2 \pm 0.2 \times 10^{9} \mathrm{~N} \mathrm{~m}^{-5} \mathrm{~s}$ at baseline, to $1.9 \pm$ $0.2 \times 10^{9} \mathrm{~N} \mathrm{~m}^{-5} \mathrm{~s}$ at 2 weeks and $7.9 \pm 0.9 \times 10^{9} \mathrm{~N} \mathrm{~m}^{-5} \mathrm{~s}$ at 4 weeks $(p<0.01$ vs control). RV-power was increased at 2 weeks, but no further increase was seen at 4 weeks (RV- power $=4.9 \pm 0.7 \mathrm{~mW}$ at baseline, $9.1 \pm 0.9 \mathrm{~mW}$ at 2 weeks, $8.3 \pm 0.7 \mathrm{~mW}$ at 4 weeks, respectively; $p<0.01$ vs controls).

Autopsy

Chronic implantation of the telemetry transmitter in both approaches did not result in any differences in organ weight as compared to control (Table 1), and no apparent pulmonary embolisms were seen.

Autopsy revealed only local fibrosis of the right ventricle near the insertion point of the catheter (ESM Fig. E-3a). In the trans-thoracic approach, animals showed normal healing of the intercostal muscles. In the trans-diaphragm approach, no herniation or dehiscence of the diaphragm were seen in any animal, and often, the liver was partially accreted to the healed wound of the diaphragm (ESM Fig. E-3b).

\section{Discussion}

The present study describes in detail two surgical techniques to monitor RV or PA pressures over time by radio-telemetry in rats. To our best knowledge, we are the first research group to describe the trans-diaphragm approach and its comparison with the previously used trans-thoracic approach. We have demonstrated that: (1) in our hands, both the trans-diaphragm as well as the transthoracic approach have satisfactory success rates, especially when considering the complexity of the procedures, (2) the time-to-recovery was significantly shorter with the trans-diaphragm approach than the trans-thoracic approach, (3) measured physiological parameters recovered fully in both methods, and there were no permanent detrimental effects on $\mathrm{RV}$, intercostal muscles or dia- 
Fig. 2 Haemodynamic changes during the development of MCT-induced pulmonary hypertension. Four weeks after the rats received $\mathrm{MCT}$, clinical signs of RV heart failure developed. In these 4 weeks, systolic pressures continued to rise, while cardiac output could not be maintained. The presence of RV remodelling was indicated by increased wall thickness and diameter. The pulmonary vascular resistance continued to rise as well, while at 4 weeks, the increase in the RV-power was inadequate to compensate for the dramatically increased afterload. Triangles connected by dashed lines: MCT-treated; squares connected by uninterrupted lines: control group; $n=3$ for both groups. All values are in mean \pm SEM, Asterisk, $p<$ 0.05 ; double asterisk, $p<0.01$ compared to control (post-hoc analyses). Please note that sometimes the icons conceal the error bars. For explanation of the abbreviations, see corresponding text
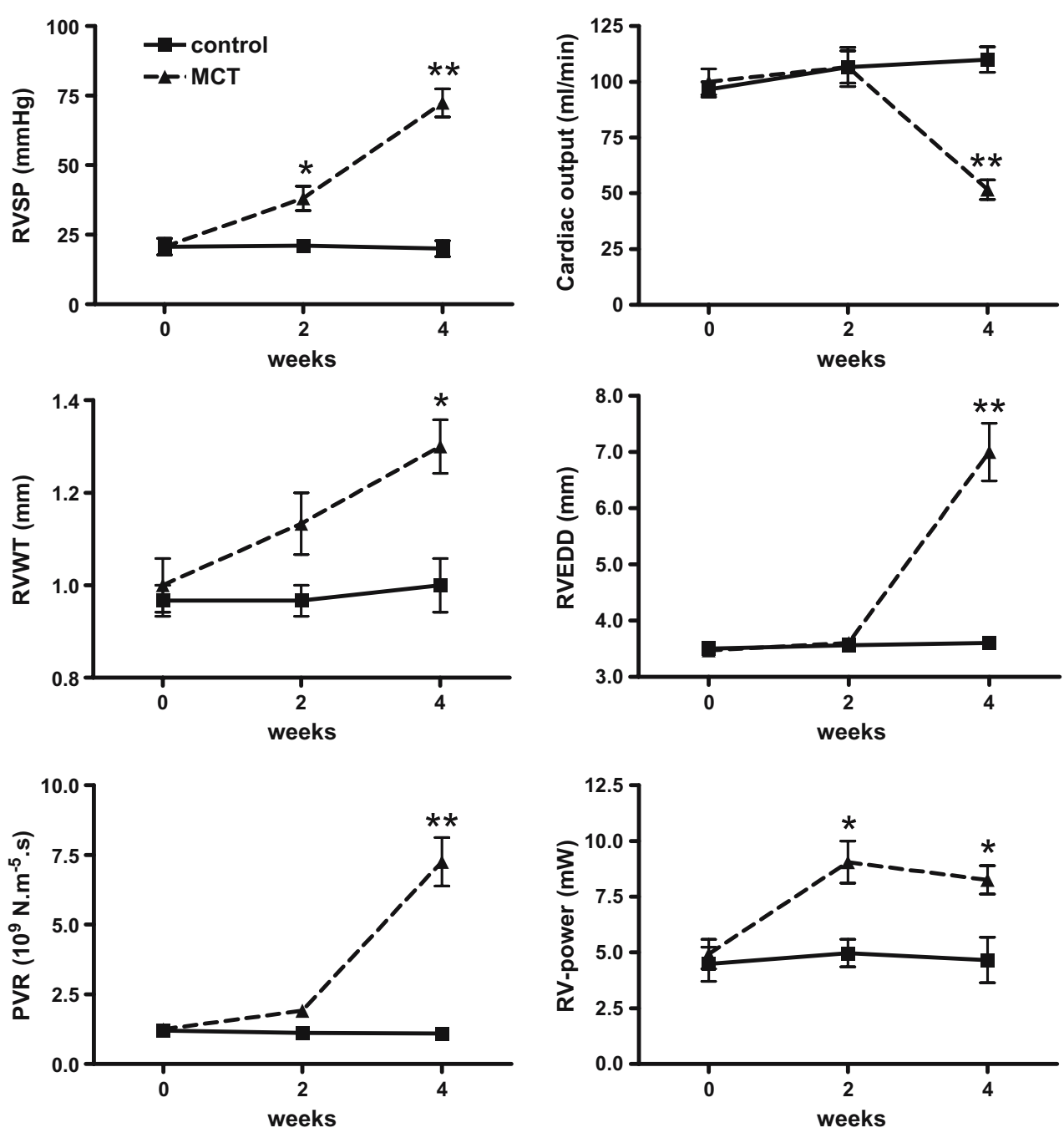

phragm, (4) using RV-telemetry, acute and chronic pressure changes in the pulmonary circulation can be readily detected and (5) RV-telemetry, in combination with echocardiography, allow thorough monitoring of pulmonary and cardiac haemodynamic changes over time.

Fully recovered animals showed normal RV structure, as expressed by RVWT and RVEDD, and normal function, as expressed by cardiac output and TAPSE. In addition, in both approaches, RV pressure normalised shortly after surgery, and autopsy revealed only minor fibrosis in the direct proximity of the catheter. Therefore, permanent detrimental effects of RV-telemetry on the RV can be excluded for both techniques. In addition, examination of other organs revealed no abnormalities, as was described by others [4]. In animals operated using the trans-diaphragm approach, macroscopic examination revealed normal wound healing of the diaphragm. Although we have not tested the strength of the healed diaphragm, others did not find significant differences between a normal (untouched) and a sutured diaphragm [7]. The natural history of small defects in the diaphragm is relatively benign, as they almost always heal spontaneously [2], especially when the diaphragm is fixated by other organs [26]. Furthermore, the observation of a normal respiratory rate seen in the recovered animals confirms that the trans-diaphragm approach is safe and a good alternative for the transthoracic approach.

In our experience, insertion of the pressure catheter into the RV and its advance further into the pulmonary artery when desired is comparatively easy to perform in the transdiaphragm approach. This relative ease of RV catheterisation is reflected in the higher success rate compared to the trans-thoracic approach and might be well explained by the more caudal and inferior direction by which the catheter enters the heart. Approaching the heart in this manner entails minimal manipulation and manoeuvring of the catheter during the process of RV catheterisation, resulting in a lower incidence of clot formation inside the catheter. This complication occurred more frequently in transthoracic-operated animals as a direct consequence of 
excessive manoeuvring and accidental squeezing of the catheter causing it to lose or displace some of its antithrombolitic gel. The strongest advocate of the transdiaphragm approach over the trans-thoracic approach, however, is the significantly faster recovery of the animals. Prompt recovery from surgery is particularly important in rats with MCT-induced $\mathrm{PH}$ because of low survival rates beyond 4 weeks after MCT injection.

In this study, we used echocardiography to validate RVtelemetry in vivo. Additionally, through the combination of pressure data, obtained by RV-telemetry, with echoDoppler flow data, we were able to study the haemodynamics of the pulmonary circulation in greater detail [23]. MCT induced pulmonary and cardiac remodelling, at the same time as PVR, RVWT and RVEDD increased. Cardiac adaptation to overcome the increasing afterload became inadequate by the fourth week of MCT injection, in such that there was no further increase in RV-power to maintain adequate cardiac output while RV systolic pressure continued to rise. Detailed studies of these observations are planned for the near future.

In the present study, we have focussed on the refinement of the RV-telemetry technique in rats, but this does not exclude its applicability in mice and other rodents. Indeed, in their recent study, Schwenke et al. [18] reported long-term monitoring of PA pressure in mice using radio-telemetry.

In conclusion, we described a new, easier-to-perform, mildly invasive trans-diaphragm-based RV-telemetry approach for long-term monitoring of PA and RV pressures in the rat model of MCT-induced PH. Our findings may be applied to improve our understanding of the disease processes involved in $\mathrm{PH}$ and develop better treatment strategies for the disease.

Acknowledgement We thank Prof. Dr. Nico Westerhof, Dr. Christa Boer and Frances de Man (VU University Medical Center, The Netherlands), Anne Brumagim and Heather Michelau (DSI, USA), Max Hardziyenka (Academic Medical Center, Amsterdam, The Netherlands), and Prof. Dr. René Remie (Solvay Pharmaceuticals, Weesp, The Netherlands) for their expert advice and discussion. M.L. Handoko is supported by the Mozaïek program/The Netherlands Organisation for Scientific Research (NWO), The Hague, The Netherlands.

Conflicts of interest None to report.

\section{References}

1. Chemla D, Castelain V, Humbert M, Hebert JL, Simonneau G, Lecarpentier Y, Herve P (2004) New formula for predicting mean pulmonary artery pressure using systolic pulmonary artery pressure. Chest 126:1313-1317

2. Gamblin TC, Wall CE Jr., Morgan JH III/, Erickson DJ, Dalton ML, Ashley DW (2004) The natural history of untreated penetrating diaphragm injury: an animal model. J Trauma 57:989-992
3. Hardziyenka M, Campian ME, de Bruin-Bon HA, Michel MC, Tan HL (2006) Sequence of echocardiographic changes during development of right ventricular failure in rat. J Am Soc Echocardiogr 19:1272-1279

4. Hess P, Clozel M, Clozel JP (1996) Telemetry monitoring of pulmonary arterial pressure in freely moving rats. J Appl Physiol 81:1027-1032

5. Hessel MH, Steendijk P, den Adel B, Schutte CI, van der Laarse A (2006) Characterization of right ventricular function after monocrotaline-induced pulmonary hypertension in the intact rat. Am J Physiol Heart Circ Physiol 291:H2424-H2430

6. Humbert M, Sitbon O, Simonneau G (2004) Treatment of pulmonary arterial hypertension. N Engl J Med 351:1425-1436

7. Kozar RA, Kaplan LJ, Cipolla J, Meija J, Haber MM (2001) Laparoscopic repair of traumatic diaphragmatic injuries. J Surg Res 97:164-171

8. Kramer K, Grimbergen JA, van der Gracht L, van Iperen DJ, Jonker RJ, Bast A (1995) The use of telemetry to record electrocardiogram and heart rate in freely swimming rats. Methods Find Exp Clin Pharmacol 17:107-112

9. Kramer K, Kinter LB (2003) Evaluation and applications of radiotelemetry in small laboratory animals. Physiol Genomics 13:197-205

10. Lang RM, Bierig M, Devereux RB, Flachskampf FA, Foster E, Pellikka PA, Picard MH, Roman MJ, Seward J, Shanewise JS, Solomon SD, Spencer KT, Sutton MS, Stewart WJ (2005) Recommendations for chamber quantification: a report from the American Society of Echocardiography's Guidelines and Standards Committee and the Chamber Quantification Writing Group, developed in conjunction with the European Association of Echocardiography, a branch of the European Society of Cardiology. J Am Soc Echocardiogr 18:1440-1463

11. Lewanowitsch T, White JM, Irvine RJ (2004) Use of radiotelemetry to evaluate respiratory depression produced by chronic methadone administration. Eur J Pharmacol 484:303-310

12. McLaughlin VV, McGoon MD (2006) Pulmonary arterial hypertension. Circulation 114:1417-1431

13. McMurtry MS, Bonnet S, Wu X, Dyck JR, Haromy A, Hashimoto K, Michelakis ED (2004) Dichloroacetate prevents and reverses pulmonary hypertension by inducing pulmonary artery smooth muscle cell apoptosis. Circ Res 95:830-840

14. Plante E, Lachance D, Roussel E, Drolet MC, Arsenault M, Couet J (2006) Impact of anesthesia on echocardiographic evaluation of systolic and diastolic function in rats. J Am Soc Echocardiogr 19:1520-1525

15. Quinones MA, Otto CM, Stoddard M, Waggoner A, Zoghbi WA (2002) Recommendations for quantification of Doppler echocardiography: a report from the Doppler Quantification Task Force of the Nomenclature and Standards Committee of the American Society of Echocardiography. J Am Soc Echocardiogr 15:167-184

16. Schermuly RT, Dony E, Ghofrani HA, Pullamsetti S, Savai R, Roth M, Sydykov A, Lai YJ, Weissmann N, Seeger W, Grimminger F (2005) Reversal of experimental pulmonary hypertension by PDGF inhibition. J Clin Invest 115:2811-2821

17. Schierok H, Markert M, Pairet M, Guth B (2000) Continuous assessment of multiple vital physiological functions in conscious freely moving rats using telemetry and a plethysmography system. J Pharmacol Toxicol Methods 43:211-217

18. Schwenke DO, Pearson JT, Mori H, Shirai M (2006) Long-term monitoring of pulmonary arterial pressure in conscious, unrestrained mice. J Pharmacol Toxicol Methods 53:277-283

19. Sebkhi A, Strange JW, Phillips SC, Wharton J, Wilkins MR (2003) Phosphodiesterase type 5 as a target for the treatment of hypoxiainduced pulmonary hypertension. Circulation 107:3230-3235

20. Stephenson R, Liao KS, Hamrahi H, Horner RL (2001) Circadian rhythms and sleep have additive effects on respiration in the rat. $\mathrm{J}$ Physiol 536:225-235 
21. Suga H (1990) Ventricular energetics. Physiol Rev 70:247-277

22. Voelkel NF, Quaife RA, Leinwand LA, Barst RJ, McGoon MD, Meldrum DR, Dupuis J, Long CS, Rubin LJ, Smart FW, Suzuki YJ, Gladwin M, Denholm EM, Gail DB (2006) Right ventricular function and failure: report of a National Heart, Lung, and Blood Institute working group on cellular and molecular mechanisms of right heart failure. Circulation 114:18831891

23. Westerhof N, Stergiopulos N, Noble MI (2005) Snapshots of hemdynamics: an aid for clinical research and graduate education, 1st edn. Springer, Heidelberg
24. Wilson DW, Segall HJ, Pan LC, Lamé MW, Estep JE, Morin D (1992) Mechanisms and pathology of monocrotaline pulmonary toxicity. Crit Rev Toxicol 22:307-325

25. Zagorski J, Debelak J, Gellar M, Watts JA, Kline JA (2003) Chemokines accumulate in the lungs of rats with severe pulmonary embolism induced by polystyrene microspheres. J Immunol 171:5529-5536

26. Zierold D, Perlstein J, Weidman ER, Wiedeman JE (2001) Penetrating trauma to the diaphragm: natural history and ultrasonographic characteristics of untreated injury in a pig model. Arch Surg 136:32-37 